\title{
Combined Relay Selection Enabled by Supervised Machine Learning
}

\author{
Shuping Dang, Member, IEEE, Jiashen Tang, Student Member, IEEE, Jun Li, Member, IEEE, \\ Miaowen Wen, Senior Member, IEEE, Salwani Abdullah, and Chengzhong Li
}

\begin{abstract}
Combined relay selection only requires two relays to forward signals transmitted on multiple subcarriers, but the optimal outage performance is almost surely achievable in the high signal-to-noise ratio (SNR) region. However, because combined relay selection involves the generation of the full set of two-relay combinations, the selection complexity of combined relay selection is much higher than that of per-subcarrier relay selection when the number of relays goes large. This drawback restricts the implementation of combined relay selection in dense networks. To overcome this drawback, we propose to enable combined relay selection by supervised machine learning (ML). Because the training procedure is off-line, the proposed implementation scheme can considerably reduce the selection complexity and the processing latency. We carry out extensive experiments on TensorFlow 2.1 over a graphics processing unit (GPU) aided computing cloud server to validate the effectiveness of the proposed scheme. The experimental results confirm that supervised ML can provide near-optimal performance with lower computing latency that well matches that provided by brute-force search and the optimal relay selection in a per-subcarrier manner.
\end{abstract}

Index Terms-Multi-carrier relay selection, supervised machine learning (ML), cooperative relaying, artificial neural network (ANN), back-propagation algorithm.

This work was supported in part by National Natural Science Foundation of China under Grant 61872102, in part by Guangxi Natural Science Foundation under Grant AD19245043, in part by Nanning Excellent Young Scientist Program under Grant RC20190201, in part by Guangxi Beibu Gulf Economic Zone Major Talent Program, in part by the International Collaborative Research Program of Guangdong Science and Technology Department under Grant No.2020A0505100061, in part by the Pearl River Nova Program of Guangzhou under Grant 201806010171, and in part by the Fundamental Research Funds for the Central Universities under Grant 2019SJ02. (Corresponding author: Jun Li.)

S. Dang was with the R\&D Center, Guangxi Huanan Communication Co., Ltd., Nanning 530007, China, and is now with Computer, Electrical and Mathematical Science and Engineering Division, King Abdullah University of Science and Technology (KAUST), Thuwal 23955-6900, Saudi Arabia (email: shuping.dang@kaust.edu.sa).

J. Tang and S. Abdullah are with Center for Artificial Intelligence, Faculty of Information Science and Technology, Universiti Kebangsaan Malaysia, 43600 Bangi, Selangor, Malaysia (e-mail: p94755@siswa.ukm.edu.my, salwani@ukm.edu.my).

$\mathrm{J}$. $\mathrm{Li}$ is with the Research Center of Intelligent Communication Engineering, School of Electronics and Communication Engineering, Guangzhou University, Guangzhou 510006, China (e-mail: lijun52018@gzhu.edu.cn).

M. Wen is with the School of Electronic and Information Engineering, South China University of Technology, Guangzhou 510641, China (e-mail: eemwwen@scut.edu.cn).

C. $\mathrm{Li}$ is with the R\&D Center, Guangxi Huanan Communication Co., Ltd., Nanning 530007, China (e-mail: chengzhong.li@hncom.ac.cn).

\section{INTRODUCTION}

$\mathbf{R}$ ELAY selection is a frequent research topic in the realm of cooperative communications and attracts tremendous attention in academia and industry [1], which can be performed in either centralized or decentralized manner [2], [3]. For multi-carrier relay systems, relay selection is of great importance and even the key to ensure network reliability with constraints on cost, complexity, and energy efficiency [4]-[6]. Combined relay selection is an efficient multi-carrier relay selection scheme capable of harvesting full diversity and almost surely achieving optimal outage performance in the high signal-to-noise ratio (SNR) region by only selecting two relays for all subcarriers, which can effectively reduce the number of relays involved in the selection process and hence the signaling overhead caused for coordinating among multiple relays [7], [8].

Despite the performance advantages of combined relay selection, when applying in dense networks where there exist a large number of relays for selection, the selection complexity becomes rather high, because it is required to generate all two-relay combinations and select the optimal combination by traversing all combinations in a per-subcarrier manner. This high selection complexity could result in unacceptable latency when implementing relay selection schemes in real time [9]. Therefore, lacking a proper enabling scheme prevents the utilization of combined relay selection in complexity-critic and latency-critic networks [10].

In this regard, we propose to enable combined relay selection by supervised machine learning (ML) with pre-processing and post-processing techniques. In the off-line phase, a reinforced artificial neural network (ANN) with a dropout layer is employed and trained by labeled data sets related to channel state information (CSI) and network topological information. In the on-line phase, the trained ANN is directly applied to identify the optimal two-relay combination and form a per-subcarrier forwarding strategy, needless to generate and traverse all two-relay combinations, which can reduce computing latency incurred. To verify the effectiveness and efficiency of the proposed enabling scheme, we present various experimental results in this paper.

The rest of this paper is organized as follows. In Section III we present the system model. The proposed supervised ML enabling framework is detailed in Section III. followed by the experiments and discussions presented in Section IV Section $\mathrm{V}$ concludes the paper. 


\section{SYSTEM MODEL}

In this paper, we suppose a generic two-hop cooperative communication network utilizing $K$ orthogonal subcarriers (the full set of subcarriers is denoted as $\mathcal{K}$, and $|\mathcal{K}|=K$ ) for information transmission. There is only a single source intending to transmit information to a single destination via $M$ clustered relays adopting the half-duplex decode-and-forward (DF) forwarding protocol (the full set of relays is denoted as $\mathcal{M}$, and $|\mathcal{M}|=M \geq 2$ ). The source selects two relays according to the combined relay selection criterion, and both selected relays decode the received signals on different subcarriers from the source and re-transmit them to the destination. Synchronization in the time and frequency domains is assumed to be perfect, and instantaneous CSI as well as the network topological information are perfectly known at both source and destination. For simplicity, equal bit and power allocation schemes are in use for all nodes in the two-hop cooperative communication network. In terms of channel models, we denote the channel power gain as $g_{i}(m, k)$, where $i \in\{1,2\}$ denoting the hop index; $m \in \mathcal{M}$ denoting the relay index, and $k \in \mathcal{K}$ denoting the subcarrier index. All channel power gains abide independent and exponential distributions with the hop-dependent mean $\mu_{i}$. By the above settings, we can have the end-to-end (E2E) SNR of the signal transmitted on the $k$ th subcarrier and forwarded by the $m$ th relay as $\gamma(m, k)=\left(P_{t} / N_{0}\right) \times \min \left\{g_{1}(m, k), g_{2}(m, k)\right\}$ [5], where $P_{t}$ and $N_{0}$ denote the uniform transmit power and the average additive white Gaussian noise (AWGN) power.

The combined relay selection scheme consists of two phases. In the first phase, the optimal two-relay combination set is identified according to the following criterion [8]:

$$
\hat{\mathcal{L}}=\arg \max _{\mathcal{L} \subseteq \mathcal{M}} \min _{k \in \mathcal{K}} \max _{l \in \mathcal{L}} \gamma(l, k),
$$

where $\mathcal{L}$ represents an arbitrary two-relay combination set with $|\mathcal{L}|=2$. After obtaining $\hat{\mathcal{L}}$, the second phase forms the persubcarrier relaying strategy, $\forall k \in \mathcal{K}$, by the criterion ${ }^{1}$

$$
\hat{l}(k)=\arg \max _{l \in \hat{\mathcal{L}}} \gamma(l, k) .
$$

To measure the reliability of multi-carrier cooperative systems in this paper, we define the system outage probability as follows [5]:

$$
\Phi\left(\gamma_{t h}\right)=\mathbb{P}\left\{\bigcup_{k \in \mathcal{K}}\left\{\gamma(\hat{l}(k), k)<\gamma_{t h}\right\}\right\},
$$

where $\mathbb{P}\{\cdot\}$ represents the probability of the random event enclosed, and $\gamma_{t h}$ is a predefined outage threshold.

From the computing perspective, we can also express the relaying strategy by an $M \times 1$ relay selection vector as $\hat{\mathbf{L}}=$ $[r(1), r(2), \ldots, r(M)]^{T}$, where $r(m)=1$ indicates that after performing the two-relay combination set selection, the $m$ th relay is selected, and $r(m)=0$, otherwise. Obviously, by 1), we have $\sum_{m=1}^{M} r(m)=2$. Because the uniform power allocation scheme is adopted, the raw input for training can be

\footnotetext{
${ }^{1}$ In this paper, we stipulate that the signal conveyed on each subcarrier will only be forwarded by a single relay for improving energy efficiency and avoiding involving combining techniques at the destination for simplicity.
}

reduced to the full CSI represented by $\{g(m, k)\}_{m \in \mathcal{M}, k \in \mathcal{K}}=$

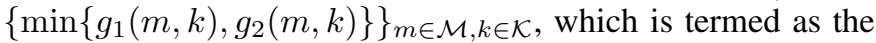
E2E equivalent channel power gain. These gains can also be organized as an $M \times K$ matrix $\mathbf{G}$. We define a labeled data set as $\{\mathbf{G}, \hat{\mathbf{L}}\}$, which can be generated by solving $[1]$ and 2 , via brute-force search for off-line training purposes.

\section{IMPLEMENTING COMBINED RELAY SELECTION BY SUPERVISED MACHINE LEARNING}

To reduce the computational complexity for performing combined relay selection and enhance the practicality, we introduce the supervised ML using the classic back-propagation algorithm to enable low-complexity combined relay selection. This is achieved by training an ANN that can return accurate subsets of selected relays. Without loss of generality, we assume that there are $D$ layers, including the input layer, the output layer, and the dropout layer, as well as $D-3$ hidden layers in a fully-connected (FC) ANN. For the $d$ th layer, there are $N_{d}$ neurons functioning as computing nodes. We denote the output from the $n_{d}$ th neuron in the $d$ th layer as $y_{d}^{n_{d}}(s)$ and the link weight between the $n_{d}$ th node and the $n_{d+1}$ th node in the adjacent layer at the $s$ th epoch as $w_{n_{d} \rightarrow n_{d+1}}(s)$. Also, the bias corresponding to the $n_{d}$ th neuron in the $d$ th layer at the $s$ th epoch is denoted as $\theta_{d}^{n_{d}}(s)$. Further supposing that there are $S$ different labeled data sets denoted as $\Lambda_{s}=\left\{\mathbf{G}_{s}, \hat{\mathbf{L}}_{s}\right\}$, $\forall s \in \mathcal{S}=\{1,2, \ldots, S\}$, we adopt the supervised ML algorithm to yield the estimated subset of selected relays $\tilde{\mathbf{L}}_{s}$ in comparison with $\hat{\mathbf{L}}_{s}$.

As the channel power gains are exponentially distributed, they can range from 0 to infinity in principle. Such a large input span renders a great challenge for any ANN to converge. To enhance the training efficiency, we design a three-stage pre-processing procedure for the input data set

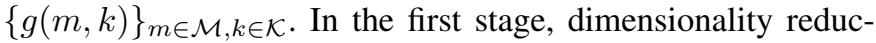
tion is performed, and the $M K \mathrm{E} 2 \mathrm{E}$ channel power gains are ranked in ascending order. By the ranked channel power gains, the original $M \times K$ matrix $\mathbf{G}_{s}$ can be rearranged as an $M K \times 1$ vector $\mathbf{c}_{s}$. In the second stage, index extraction is carried out, and the indexes of the entries in $\mathbf{c}_{s}$ are extracted to form another $M K \times 1$ vector $\mathbf{u}_{s}$. In the final stage, normalization is conducted over vector $\mathbf{u}_{s}$, and the indexes in $\mathbf{u}_{s}$ are normalized, which yields an $M K \times 1$ input vector $\mathbf{v}_{s}=\left[v_{1}(s), v_{2}(s), \ldots, v_{M K}(s)\right]^{T}=\mathbf{u}_{s} / M K$. Here, we make full use of the domain knowledge that the outcome of combined relay selection is only dependent on the ranks of channel power gains instead of the absolute values. After performing this three-stage pre-processing procedure, we restrict the input span to be $(0,1]$. Accordingly, the neuron output in the input layer is $y_{1}^{n_{1}}(s)=v_{n_{1}}(s), 1 \leq n_{1} \leq M K$, which requires neither processing nor manipulation. For the hidden layers and the output layer, the output of the $n_{d}$ th neuron in the $d$ th layer is given by

$$
y_{d}^{n_{d}}(s)=\operatorname{ReLU}\left(\sum_{n_{d-1}=1}^{N_{d-1}} w_{n_{d-1} \rightarrow n_{d}}(s) y_{d-1}^{n_{d-1}}(s)+\theta_{d}^{n_{d}}(s)\right) \text {, }
$$


where $\operatorname{ReLU}(\cdot)=\max (0, \cdot)$ is the rectified linear unit (ReLU) of the enclosed argument.

Before training, we need to initialize the ANN either in a random way or by loading initialization setup files, which refer to the stochastic and deterministic initialization methods, respectively. The entire training procedure can be classified into the forward-propagation and back-propagation phases. In the forward-propagation phase, the data sets will be generated by slicing the entire training data according to a preset batch size. Then, the per-batch training is carried out to yield the output of the ANN, which will be compared with data label to produce a loss matrix and subsequently a gradient matrix. The gradient matrix is then processed by a chosen optimizer to determine how network parameters, e.g., link weights and neural biases, will be updated ${ }^{2}$ Finally, the learning process will be terminated if all termination conditions are met. Otherwise, the learning process will be continued on an iterative basis. Once the iterative learning process is stopped, the resultant ANN with updated link weights and biases is said to be trained.

The overfitting problem is the major concern of employing a trained ANN with randomly generated samples. To mitigate this problem, in our experiments, we stipulate an early-stop mechanism and add a dropout layer prior to the output layer ${ }^{3}$ In particular, after completing the training for each epoch, the mean squared error (MSE) of the current epoch is compared with all MSEs in the previous epochs to check whether the training performance is improved or not (definition and discussion regarding the MSE will be given later in this section). If the MSE has not decreased for a preset number of continuous epochs, the current training round is terminated. As the second line of defense, the dropout layer is added so that the outputs of the last hidden layer could be randomly set to zero with a preset probability ${ }^{4} \rho$. On the other hand, the remaining non-zero outputs from the last hidden layer are rescaled by a multiplier $\eta=1 /(1-\rho)$. In this way, the training parameters are less likely to be trapped in overfitting regions.

In addition, the outputs of the ANN are organized by a $K \times 1$ vector $\tilde{\mathbf{q}}_{s}$ approximating the normalized indexes of relays forwarding all $K$ subcarriers. To interpret the outputs of the ANN, we also need to employ a three-stage inverse postprocessing procedure. Specifically, the entries in $\tilde{\mathbf{q}}_{s}$ multiply $M K$ to give an intermediate $K \times 1$ vector $\tilde{\mathbf{p}}_{s}$. Then, the entries of $\tilde{\mathbf{p}}_{s}$ are rounded to the nearest integers bounded by $M K$. Note that the $K \times 1$ vector of these rounded integers is denoted as $\tilde{\mathbf{f}}_{s}$, indicating the approximate indexes of the selected relays forwarding all $K$ subcarriers. As there exists a bijective mapping relation between $\tilde{\mathbf{f}}_{s}$ and $\tilde{\mathbf{L}}_{s}$, we can obtain the estimated subset of selected relays $\tilde{\mathbf{L}}_{s}$ by $\tilde{\mathbf{f}}_{s}$. From above descriptions, it is obvious that $N_{1}=M K$ and

\footnotetext{
${ }^{2}$ The updating method of the network parameters in the following experiments is the classic back-propagation algorithm as detailed in [11], which has also been applied to enable two basic multi-carrier relay selection schemes in [12]. Interested readers can refer to the technical details therein.

${ }^{3}$ The early-stop mechanism can be supported by the various callback functions provided by Keras.

${ }^{4}$ Note that, the drop-out behaviors regarding the $N_{D-2}$ outputs from the last hidden layer are mutually independent. Also, the dropout layer only functions in the training iterations. After solidifying the ANN, the drop-out layer is automatically nullified by setting the drop-out probability to zero.
}

$N_{D}=K$ corresponding to the numbers of entries of $\mathbf{v}_{s}$ and $\tilde{\mathbf{q}}_{s}$, respectively.

To measure the training performance so as to check whether an ANN has been well trained or not, we can easily construct a vector of optimal indexes of the selected relays forwarding all $K$ subcarriers, denoted as $\hat{\mathbf{f}}_{s}$, from $\hat{\mathbf{L}}_{s}$. Then, we define the MSE over a number of continuous epochs to measure the training performance: $\mathrm{MSE}=\mathbb{E}\left\{\left\|\hat{\mathbf{f}}_{s}-\tilde{\mathbf{f}}_{s}\right\|_{2}^{2}\right\}$, where $\mathbb{E}\{\cdot\}$ denotes the average of the random variable enclosed, and $\|\cdot\|_{2}$ denotes the two-norm of the enclosed matrix. Meanwhile, we also define the network accuracy by $\mathrm{ACC}=\frac{1}{\Xi} \sum_{s=1}^{\Xi} \mathbb{I}\left\{\tilde{\mathbf{L}}_{s}=\right.$ $\left.\hat{\mathbf{L}}_{s}\right\}$, where $\Xi \leq S$ is the actual number of running epoch 5 and $\mathbb{I}\{\cdot\}$ is the Iverson bracket.

\section{EXPERIMENTS AND KEY OBSERVATIONS}

\section{A. Experimental Setups}

To verify the effectiveness of the proposed enabling scheme of combined relay selection, we carried out the experiments on TensorFlow 2.1 over a graphics processing unit (GPU) aided computing cloud server (GN10X by Tencent Cloud: https://intl.cloud.tencent.com/). The main development and debugging environment is PyCharm IDE. Occasionally, Vim in a Linux server is used to adjust trivial parameters. The operating system is Linux Centos 7.6 64-bit. We utilize Anaconda to construct and maintain the operating environment by installing the packages of numpy and tensorflow $2.1 \mathrm{gpu}$ as well as dependencies. After installing these packages and debugging the operating environment, we uploaded the local source codes to the cloud server and remotely operated them.

The main architecture of the operating program consists of three parts: constructing/loading ANN model, training (including generating training data, conducting iterative training, and evaluating performance), and checking termination conditions. In the source codes, there are three files named nn_relay.py, nn_relay_util.py, and nn_relay_verify.py, taking the responsibility of the corresponding functions. nn_relay.py is the main file of the source codes and defines the core process and the structure of the ANN. nn_relay_util.py defines a number of general-purpose functions that are frequently called over the entire operating process. nn_relay_verify.py is used to inspect the training outputs, check the termination conditions, and evaluate performance of the ANN. The main architecture of the operating program with four types of necessary information for training can be depicted as a flowchart in Fig. 1 .

For simplicity, the cooperative network is configured to be spatially static with $M=8$ relays for selection, and $K=2$ subcarriers are available for conveying information to transmit. We normalize $P_{t} / N_{0}=1$ and $\gamma_{t h}=1$, and let $\mu_{1}=1$ and $\mu_{2}=2$ to capture the spatial heterogeneity of multi-hop cooperative systems. We initialize the learning rate $\alpha=0.001$ and adapt it by a dynamic mechanism depending on the training accuracy. In particular, the program regularly checks the training accuracy and multiplies the current learning rate

\footnotetext{
${ }^{5}$ Because of the early-stop mechanism adopted, the actual number of running epochs $\Xi$ can be less than $S$ when encountering overfitting.
} 


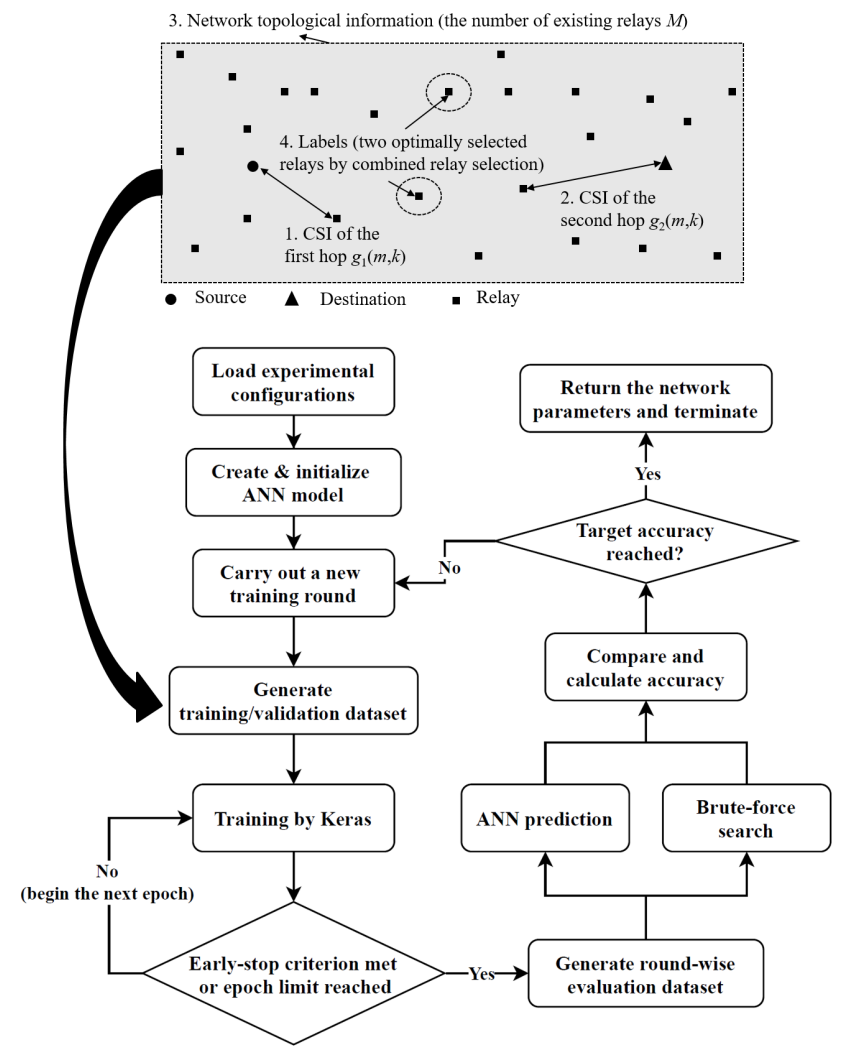

Fig. 1: Flowchart of the operating program with four types of necessary information for training an ANN to enable combined relay selection: 1) CSI of the first hop (source-relay link); CSI of the second hop (relay-destination link); 3) network topological information (how many relays exist in the network for selection); 4) labels (which two relays are optimally selected).

by 0.1 when the training accuracy has not been improved for the last 20 rounds. All link weights are initialized by the embedded Glorot normal initializer of Keras. The Glorot normal initializer adopts a stochastic initialization method by drawing samples from a truncated normal distribution. All neural biases are initialized to zero in the following experiments for simplicity. The instantaneous channel power gains are randomly generated by the random seed of exponential distribution embedded on numpy. For each training round, it is configured in our experiments that there are 2000 epochs at most, i.e., $S=2000$. The preset dropout probability is configured to be $\rho=0.1$. To check the generalization ability of the trained ANN, we split all data sets into the training and validation categories. The former is used to train the ANN and fit the actual mapping relation between input and output, while the latter is used to provide a reference for checking the generalization ability of the trained ANN. Source codes of all experiments herein can be found on Github through the link: https://github.com/DnzJS/BPANN_RelaySelection.

In the following experiments, we specify the complete ANN structure as in Table I In particular, there are eleven layers, including the input, output, and dropout layers (labeled as \#0x), as well as eight hidden layers (labeled as HxX). The complete ANN structure is designed following the dual-funnel shape, aiming at mitigating the bottleneck effect of the hidden layers. Also, to investigate the impacts of different ANN structures on
TABLE I: Specification of the complete ANN structure.

\begin{tabular}{c|c|c|c|c}
\hline $\begin{array}{c}\text { Layer } \\
\text { ID }\end{array}$ & $\begin{array}{c}\text { Layer } \\
\text { type }\end{array}$ & $\begin{array}{c}\text { Neuron } \\
\text { Number }\end{array}$ & $\begin{array}{c}\text { Activation } \\
\text { function }\end{array}$ & Initializer \\
\hline \hline$\# 01$ & FC & $M K$ & ReLU & Glorot_normal \\
\hline H1 & FC & $M^{2} K$ & ReLU & Glorot_normal \\
\hline H2A & FC & $2 M^{2} K$ & ReLU & Glorot_normal \\
\hline H4A & FC & $4 M^{2} K$ & ReLU & Glorot_normal \\
\hline H8A & FC & $8 M^{2} K$ & ReLU & Glorot_normal \\
\hline H16 & FC & $16 M^{2} K$ & ReLU & Glorot_normal \\
\hline H8B & FC & $8 M^{2} K$ & ReLU & Glorot_normal \\
\hline H4B & FC & $4 M^{2} K$ & ReLU & Glorot_normal \\
\hline H2B & FC & $2 M^{2} K$ & ReLU & Glorot_normal \\
\hline$\# 02$ & Dropout & N/A & N/A & N/A \\
\hline$\# 03$ & FC & $K$ & Linear & Glorot_normal \\
\hline
\end{tabular}

the training performance, we create a set of ANN structures in a slice-and-reconstruct manner from the complete ANN structure. That is, the complete ANN structure is first sliced into eleven layers, and then different layers are selected to reconstruct new ANN structures. In this way, the reconstructed ANNs can be regarded as substructures of the complete ANN structure. We generate and study eight representative ANN structures in the following experiments, which are designed by slicing and reconstruction and numbered as follows:

- Structure 0: \#01,\#02,\#03

- Structure 1: \#01,H1,\#02,\#03

- Structure 1_2: \#01,H1,H2A,H2B,\#02,\#03

- Structure 1_4: \#01,H1,H2A,H4A,H2B,\#02,\#03

- Structure 2_4: \#01,H1,H2A,H4A,H4B,H2B,\#02,\#03

- Structure 1_8: \#01,H1,H2A,H4A,H8B,H4B,H2B,\#02,\#03

- Structure 2_8: \#01,H1,H2A,H4A,H8A,H8B,H4B,H2B,\#02,\#03

- Structure 16: \#01,H1,H2A,H4A,H8A,H16,H8B,H4B,H2B,\#02,\#03

\section{B. Discussion of the Experimental Results}

In this subsection, we focus on the two key metrics of training performance, i.e., the MSE and the network accuracy, and present the experimental results corresponding to different ANN structures and optimizers in Fig. 22 and Fig. 3, respectively. Different optimizers have different algorithms to adjust the parameters in the ANN model. We adopt several classic optimizers, including Adam, SGD, Adadelta, and Nadam for comparison. From Fig. 2, it is clear that all ANN structures are able to improve the training performance in different scales. However, the training performance of oversimple ANN structures, e.g., Structures 0,1 , and $1 \_2$, is relatively poor. On the other hand, a more sophisticated ANN structure does not necessarily lead to fast convergence. For example, Structure $2 \_8$ is more complicated than Structures 1_4, 2_4, and 1_8, but is much slower to converge. From Fig. 3, although all optimizers can improve the ANN training performance, Adam is most efficient for the combined relay selection problem.

To be insightful, we also investigate the training process on the epoch level and illustrate the variation in MSE for both training and validation data sets in Fig. 4. To ensure sufficient expressive power and fast convergence, we adopt Structure 16 and Adam as the ANN structure and the optimizer, respectively. As shown in this figure, except for the initial overshoots, the training performance keeps getting better for an increasing 

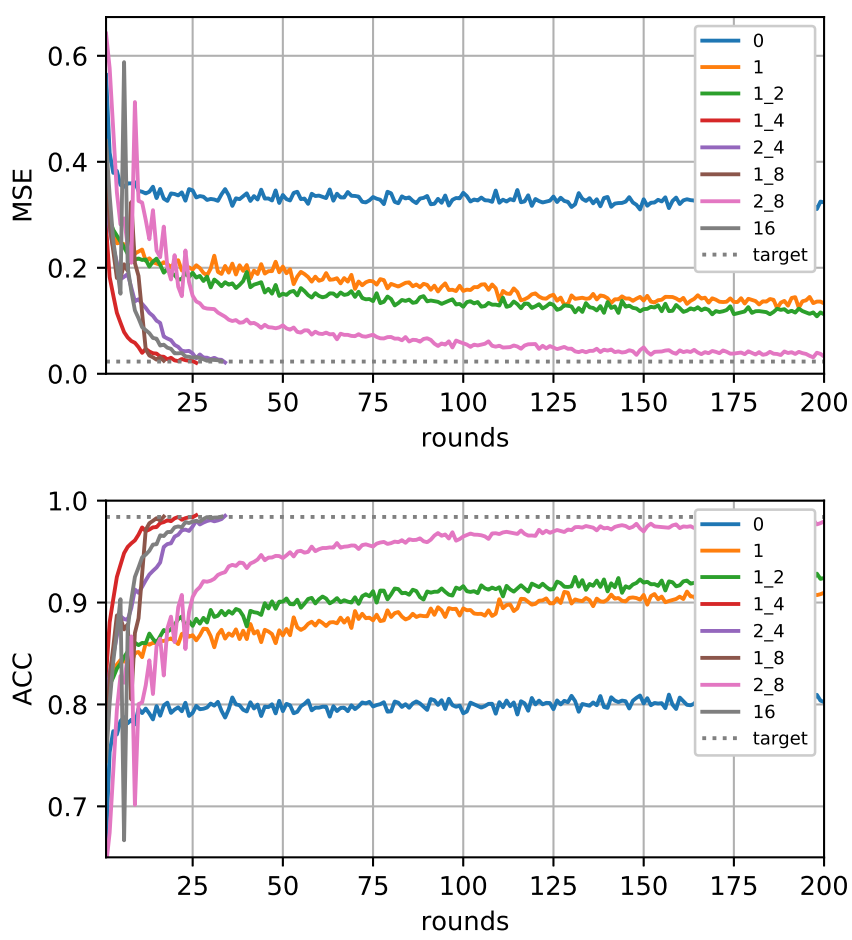

Fig. 2: MSE and network accuracy for different ANN structures by slicing and reconstruction when Adam is adopted as the optimizer.
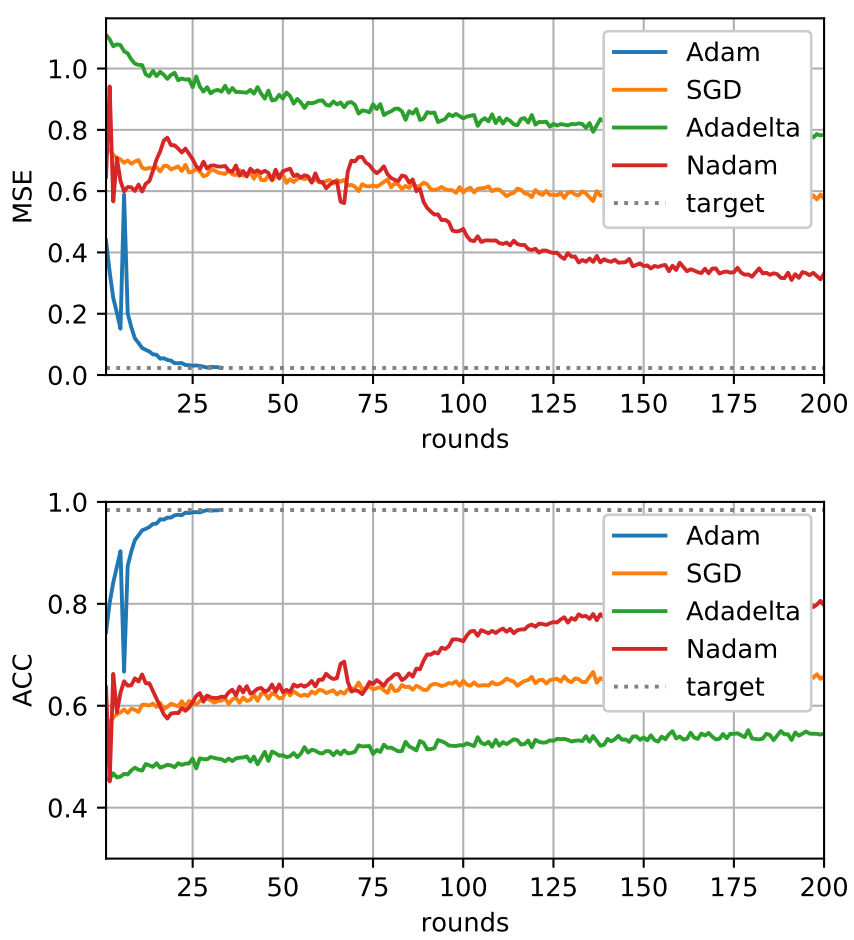

Fig. 3: MSE and network accuracy for different optimizers upon Structure 16 (i.e., the complete ANN structure).

number of epochs within a single round. However, in a new round, because of data set refreshes, the training performance becomes worse immediately but soon gets back to the normal level. Frequent data set refreshes can help ANN get rid of

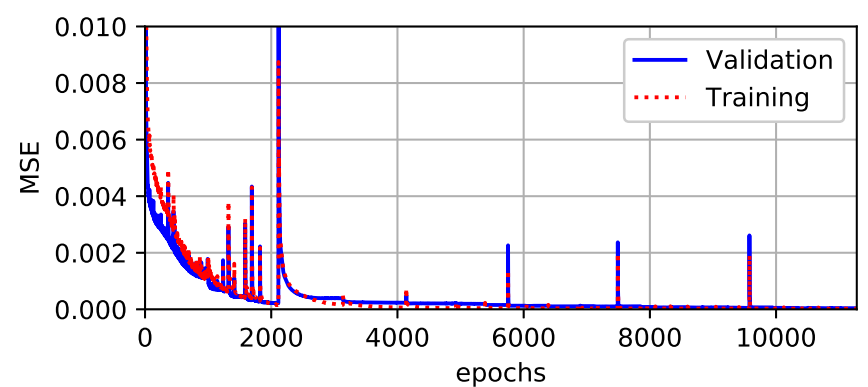

Fig. 4: MSE for multiple training and validation data sets on the epoch level.

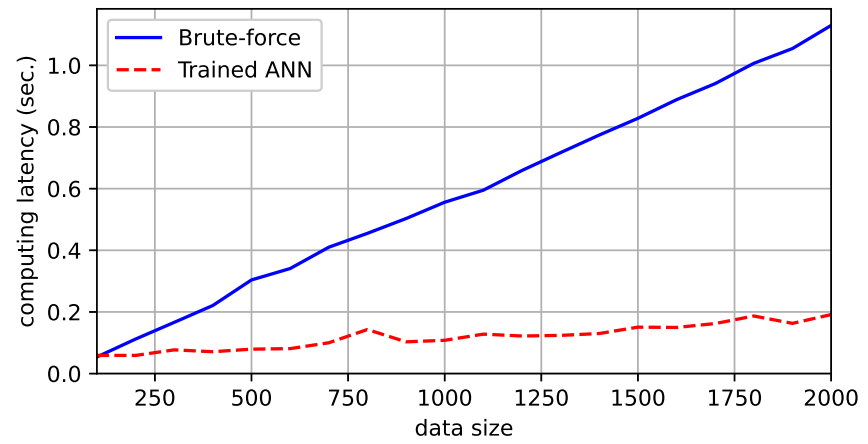

Fig. 5: Computing latency versus data size upon Structure 1_4.

local saddle points, and more importantly, explores a much larger search space. As a result, the overfitting problem can be mitigated. As the surges in MSE caused by data set refreshes also get lower after several rounds, these results demonstrate the strong robustness and transferability of the trained ANN.

To quantify the computing advantage of supervised ML, the computing latency of both trained ANN and brute-force search is shown and compared in Fig. 5, where Structure 1_4 is adopted for neural computing due to its simplicity. The data size quantifies the data volume of a single batch, signifying the capability of feeding CSI back for training purposes. From this figure, the computing latency of trained ANN is much smaller than that of brute-force search, except for batches with very small data sizes, which implies a considerable computing advantage brought by supervised ML for the combined relay selection problem.

Apart from training performance, communication performance is of great importance for validating the proposed supervised ML enabling scheme. We compare the outage performance of the multi-carrier relay systems relying on the relays selected by the trained ANN with those of brute-force search, random selection, bulk selection, and per-subcarrier selection ${ }^{6}$ (the optimal selection scheme) in Fig. 6 As demonstrated in this figure, the outage performance yielded by the proposed scheme exactly matches that of brute-force search and is much better than those of random and bulk relay selection schemes. Because of the equivalence principle, combined relay selection

\footnotetext{
${ }^{6}$ Technical details of the bulk and per-subcarrier relay selection schemes can be found in [13], which represent the two extreme cases of the complexityperformance trade-off of multi-carrier relay selection.
} 


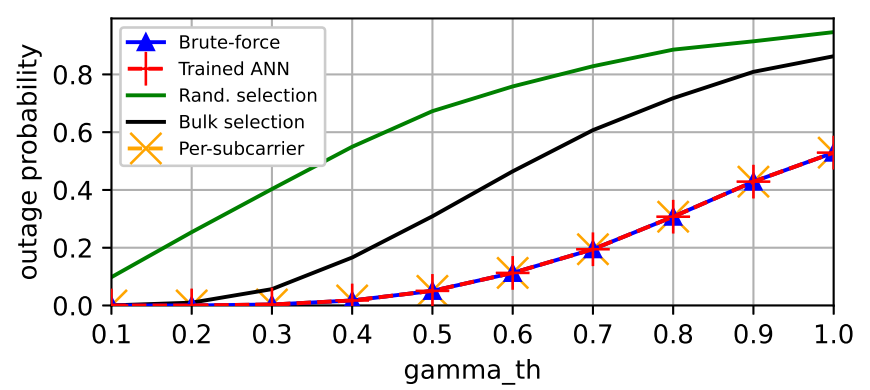

Fig. 6: Outage performance versus outage threshold $\gamma_{t h}$ for different relay selection schemes.

can yield almost the same outage performance as the optimal per-subcarrier relay selection for small $\gamma_{t h}$. This verifies the effectiveness and communication performance superiority of the proposed supervised ML enabling scheme for combined relay selection.

Through the key observations presented, we can verify the effectiveness of the supervised ML based enabling scheme for combined relay selection from both computing and communications perspectives. The proposed enabling scheme well fits the complexity-critic and latency-critic wireless applications in the Internet of Things (IoT) and machine-type communication (MTC) networks. Meanwhile, it is worth noting that although we assumed that the transmission environment is time-invariant with fixed statistical parameters, the aging problem of trained ANNs in dynamical transmission environments of the time-varying nature can be settled by introducing an online learning module [14], [15]. Equipped with such an upgrading module, either the source or the destination will occasionally send real-time unlabeled samples to a centralized server for producing labels and updating the trained ANN. Also, although we studied the enabling scheme of combined relay selection under the assumption of perfect centralized coordination among relays for simplicity, with the progress of distributed learning architectures and techniques [16], the enabling scheme can also be applied to distributed relay networks with the support of timers mounted on relays [17].

\section{CONCLUSION}

In this paper, we proposed a supervised ML enabling scheme to enable combined relay selection in multi-carrier cooperative networks to overcome the drawback of high computing latency. We carried out extensive experiments on TensorFlow 2.1 over a GPU aided computing cloud server to verify the effectiveness and efficiency of the enabling scheme. By employing the proposed enabling scheme, the application scenarios of combined relay selection are expected to be extended to complexity-critic and latency-critic networks. Moreover, this work also provides a new vision to resolve complex optimization problems in wireless communications by supervised ML and reinforced ANN. Several computing techniques, e.g., the three-stage pre-processing and postprocessing, as well as the early-stop mechanism and the involvement of a dropout layer, can be easily extended to other similar optimization problems for training performance enhancement.

\section{REFERENCES}

[1] Y. Yang, H. Hu, J. Xu, and G. Mao, "Relay technologies for WiMax and LTE-advanced mobile systems," IEEE Commun. Mag., vol. 47, no. 10, pp. 100-105, Oct. 2009.

[2] G. C. Alexandropoulos, A. Papadogiannis, and K. Berberidis, "Performance analysis of cooperative networks with relay selection over Nakagami- $m$ fading channels," IEEE Signal Process. Lett., vol. 17, no. 5, pp. 441-444, May 2010.

[3] A. Bletsas, A. Lippnian, and D. P. Reed, "A simple distributed method for relay selection in cooperative diversity wireless networks, based on reciprocity and channel measurements," in Proc. IEEE VTC, vol. 3, Stockholm, Sweden, Dec 2005, pp. 1484-1488.

[4] K. Sung, Y. . P. Hong, and C. Chao, "Resource allocation and partner selection for cooperative multicarrier systems," IEEE Trans. Veh. Technol., vol. 60, no. 7, pp. 3228-3240, Sept. 2011.

[5] W. Yang and Y. Cai, "On the performance of the block-based selective OFDM decode-and-forward relaying scheme for 4G mobile communication systems," J. Commun. Netw., vol. 13, no. 1, pp. 56-62, Feb. 2011.

[6] S. Dang, G. Chen, and J. P. Coon, "Multicarrier relay selection for fullduplex relay-assisted OFDM D2D systems," IEEE Trans. Veh. Technol., vol. 67, no. 8, pp. 7204-7218, Aug. 2018.

[7] Y. Li, W. Wang, and F. Zheng, "Combined bulk and per-tone relay selection in cooperative OFDM systems," in Proc. IEEE ICCC, Beijing, China, Aug. 2012, pp. 487-491.

[8] S. Dang, J. P. Coon, and G. Chen, "An equivalence principle for OFDMbased combined bulk/per-subcarrier relay selection over equally spatially correlated channels," IEEE Trans. Veh. Technol., vol. 66, no. 1, pp. 122133, Jan. 2017

[9] V. Shah, N. B. Mehta, and R. Yim, "Optimal timer based selection schemes," IEEE Trans. on Commun., vol. 58, no. 6, pp. 1814-1823, June 2010.

[10] Z. Zhao, M. Peng, Y. Ma, Y. Li, C. Yang, and Y. Wu, "Cooperative transmissions in 5G large-scale relay systems: How to keep a balance between performance and complexity?" J. Signal Process. Syst., vol. 83, no. 2, pp. 207-223, May 2016.

[11] M. Negnevitsky, Artificial Intelligence: A Guide to Intelligent Systems. Addison-Wesley, 2005.

[12] S. Dang, M. Wen, S. Mumtaz, J. Li, and C. Li, "Enabling multi-carrier relay selection by sensing fusion and cascaded ANN for intelligent vehicular communications," IEEE Sens. J., 2020 (early access article).

[13] S. Dang, J. P. Coon, and G. Chen, "Outage performance of two-hop OFDM systems with spatially random decode-and-forward relays," IEEE Access, vol. 5, pp. 27 514-27 524, Nov. 2017.

[14] M. Chen, U. Challita, W. Saad, C. Yin, and M. Debbah, "Artificial neural networks-based machine learning for wireless networks: A tutorial," IEEE Commun. Surv. Tutor, vol. 21, no. 4, pp. 3039-3071, Fourthquarter 2019.

[15] E. Bjornson and P. Giselsson, "Two applications of deep learning in the physical layer of communication systems [lecture notes]," IEEE Signal Process. Mag., vol. 37, no. 5, pp. 134-140, Sept. 2020.

[16] M. Chen, Z. Yang, W. Saad, C. Yin, H. V. Poor, and S. Cui, "A joint learning and communications framework for federated learning over wireless networks," IEEE Trans. on Wireless Commun., 2020 (early access article).

[17] T. Fu, S. Dang, L. Wu, and M. Wen, "Combined relay selection in spatially random networks," IEEE Syst. J., 2020 (early access article). 